\title{
Change in the cause of inpatient mortality after arthroplasty: a retrospective study
}

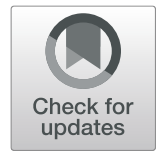

\author{
Yuzhi Zuo, Jin Lin, Jin Jin, Wenwei Qian, Guixing Qiu and Xisheng Weng (D)
}

\begin{abstract}
Background: Although arthroplasty has been proved to be a safe and effective procedure, data regarding inpatient mortality rates associated with arthroplasty in China is unclear. We aimed to investigate the inpatient mortality rate after arthroplasty and the determinants of mortality at our center to ensure improved perioperative management.

Methods: This retrospective study included all patients who underwent arthroplasty at our center. Clinical data of mortality patients were collected. The incidence and the causes of inpatient mortality after arthroplasty were analyzed.

Results: A total of 4176 total knee arthroplasties, 2164 total hip arthroplasties, and 1031 femoral head replacements were performed. A rapid growth in surgery volume was observed, and more than $50 \%$ of the surgeries were performed in the last 5 years. The overall inpatient mortality rate is $0.3 \%$; however, the mortality rate even decreased in the last 5 years. The cause of death changed over time. Pneumonia has become the leading cause of death in the past 5 years instead of cardiovascular complications.

Conclusions: Arthroplasty is a safe and effective procedure associated with a relatively low inpatient mortality in China. And inpatient mortality does not increase as the growing surgery volume due to improvement of perioperative management. However, patients presenting with risk factors and those undergoing non-elective procedures demonstrated a relatively high incidence of postoperative complications, particularly pneumonia.
\end{abstract}

Keywords: Joint arthroplasty, Inpatient mortality, Pneumonia, Cardiovascular disease

\section{Background}

Arthroplasty including total hip/knee arthroplasty (THA/TKA) and femoral head replacement (FHR) is safe and effective to treat patients with arthropathy like severe osteoarthritis, rheumatoid arthritis, and ankylosing spondylitis, as well as patients presenting with femoral neck fracture. It can significantly relieve the joint pain, restore the joint function, and improve the quality of life [1]. Over the last few decades, advances in surgical technology, prosthetic materials, enhanced recovery therapy strategy, and perioperative management have led to a significant reduction in surgical mortality rates. Owing to advances in the medical field and a rapidly growing aging population, indications of the arthroplasty have broadened. As older patients and those with more comorbidities are undergoing arthroplasties [2], the postoperative complications especially mortality rate

\footnotetext{
* Correspondence: Drwengxish@163.com

Department of Orthopedic Surgery, Peking Union Medical College Hospital, Peking Union Medical College and Chinese Academy of Medical Sciences, No.1 Shuaifuyuan, Beijing 100730, China
}

remains a serious concern. Reportedly, the inpatient, the 30-day, and the 90- day mortality rates after elective TKA are $0.09-0.13 \%, 0.2-0.3 \%$, and $0.4-0.7 \%$, respectively; whereas those after elective THA are $0.18-0.19 \%$, $0.2-0.95 \%$, and $0.5-0.7 \%$ [3-7], respectively. Fractures increase the risk of postoperative death and the 90-day mortality rate following THA or FHR performed to treat femoral neck fracture is approximately $9.3 \%$ [8].

To our knowledge, data regarding inpatient mortality rates associated with arthroplasty in China is unclear. We aimed to investigate the inpatient mortality rate after arthroplasty and to investigate the determinants of mortality at our center to ensure improved perioperative management.

\section{Methods}

This retrospective study included all patients who underwent arthroplasty at our center. The incidence and the causes of inpatient mortality after arthroplasty were analyzed. Clinical data of mortality patients including demographic characteristics, preoperative diagnosis, co-

(c) The Author(s). 2019 Open Access This article is distributed under the terms of the Creative Commons Attribution 4.0 International License (http://creativecommons.org/licenses/by/4.0/), which permits unrestricted use, distribution, and 
morbidities, American Society of Anesthesiologists (ASA) classification, anesthesiologic method, surgery, postoperative complications, cause of death, and hospital length of stay (LOS) were collected. This study was approved by the Ethics Committee of our hospital.

\section{Results}

A total of 4176 TKAs, 2164 THAs, and 1031 FHRs were performed at our center between 1982 and 2017. Among them, 4054 were elective primary TKAs, 1635 were elective primary THAs, 65 were elective FHRs, and 1194 THAs/FHRs were performed to treat femoral neck fracture. A rapid growth in surgery volume was observed and more than $50 \%$ of the surgeries were performed in the last 5 years, with a shorter LOS (Fig. 1).

Overall, 24 patients died during hospitalization owing to postoperative complications after arthroplasty with an overall inpatient mortality rate of $0.3 \%$ (24/7371). Four patients died after elective TKA with an inpatient mortality rate of approximately $0.1 \%(4 / 4054)$, and 1 patient died after elective THA with an inpatient mortality rate of approximately $0.06 \%(1 / 1635)$. The remaining patients died after THA/FHR secondary to femoral neck fracture, with an inpatient mortality rate of approximately $1.6 \%$ (19/1194). Medical records were missing in 4 patients (two elective TKAs, one elective THA, and one FHR after femoral neck fracture); thus, we analyzed the data obtained from the remaining 20 patients ( 2 elective and 18 non-elective patients) (Table 1 ).

Both patients who died after the elective procedures died of cerebral infarction (CI). One had a history of stroke, and the other had a history of atrial fibrillation. These patients developed CI intraoperatively and on post operation day (POD) 1, respectively.

The 18 patients who underwent non-elective procedures developed 53 postoperative complications including cardiovascular disease, pneumonia, cerebrovascular disease, septic shock, and others such as acute renal failure and local wound infection (Table 2). Among them, pneumonia and cardiovascular disease were the most lethal complications. The cause of death and inpatient mortality rate changed over time. Prior to 2012, cardiovascular complications were the main causes of death; however, after 2012, pneumonia was the leading cause of death (Table 3).

Seven patients died of postoperative septic shock. Six of them were diagnosed with pneumonia and 1 with abdominal infection. Four of these patients died within the last 5 years. The mean age of patients with pneumonia was 85 . Although no patient reported a history of smoking, 3 patients reported preoperative fever and cough. These symptoms were relieved preoperatively following the administration of antibiotics; however, patients with pneumonia deteriorated postoperatively. The other 3 patients with pneumonia were asymptomatic but showed risk factors for pneumonia. One patient presented with delirium and drowsiness preoperatively and could not cooperate with coughing and pulmonary function exercises. He developed pneumonia on POD 1. The second patient developed heart failure, bleeding, and renal failure postoperatively, which led to prolonged immobility. He developed pneumonia after he was admitted to the intensive care unit (ICU). The third patient was a 91year-old woman who developed pneumonia on POD 13. The patient diagnosed with abdominal infection developed infectious diarrhea secondary to food contamination and showed diffuse peritonitis postoperatively.

Eight patients died of postoperative cardiovascular complications (only 1 patient died in the last 5 years). Three patients presented with acute myocardial infarction (AMI). One reported a history of coronary heart disease (CHD) and was diagnosed as AMI on POD 1, whereas the other 2 denied a history of CHD but reported a history of hypertension and diabetes and were diagnosed with AMI on POD 6 and 7, respectively. Two patients showed severe arrhythmia. One had a history of sinus arrhythmia and developed sudden cardiac arrest intraoperatively, whereas the other had a history of hypertension, CHD, old myocardial infarction, and cardiac
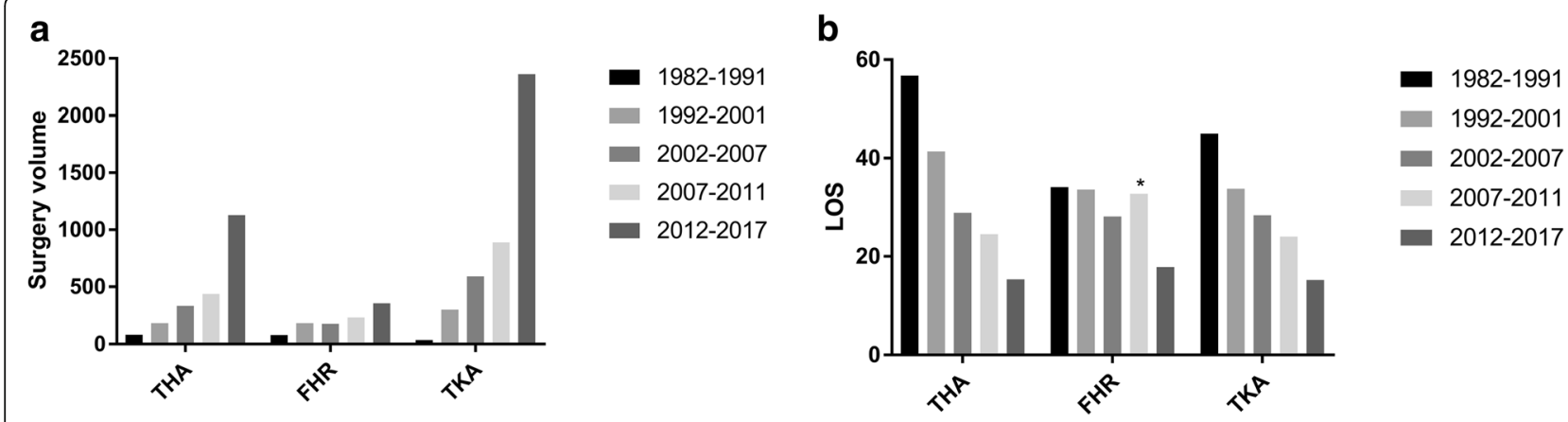

Fig. 1 a, $\mathbf{b}$ Trend of surgery volume and hospital length of stay over the last decades. Surgery volume increased rapidly and the LOS decreased significantly especially in the last 5 years. The asterisk indicates there were two patients who stay in hospital for a very long time (685 days and 1083 days) after FHR because of complications during 2007-2011 period. The LOS is shorter than 2002-2007 period after excluding these two patients 
Table 1 Demographics of mortality patients after joint arthroplasty

\begin{tabular}{|c|c|c|c|}
\hline & TKA $(n=2)$ & THA/FHR $(n=18)$ & Total $(n=20)$ \\
\hline Age (year, mean \pm SD) & $75 \pm 4.24$ & $82.28 \pm 9.59$ & $81.55 \pm .39$ \\
\hline Gender (male/female) & $0 / 2$ & $7 / 11$ & $7 / 13$ \\
\hline \multicolumn{4}{|l|}{ Preoperative diagnosis } \\
\hline Osteoarthritis & 2 & 0 & 2 \\
\hline Femoral neck fracture & / & 18 & 18 \\
\hline \multicolumn{4}{|l|}{ Comorbidity } \\
\hline \multicolumn{4}{|l|}{ Cardiovascular system } \\
\hline Hypertension & 2 & 9 & 11 \\
\hline$C H D^{a}$ & 0 & 4 & 4 \\
\hline Old $\mathrm{Ml}^{\mathrm{a}}$ & 0 & 2 & 2 \\
\hline Heart failure & 0 & 1 & 1 \\
\hline Arrhythmia & 1 & 5 & 6 \\
\hline \multicolumn{4}{|l|}{ Cerebrovascular system } \\
\hline Old Cla & 1 & 4 & 5 \\
\hline$T I A^{a}$ & 0 & 1 & 1 \\
\hline \multicolumn{4}{|l|}{ Perivascular disease } \\
\hline Arterial atherosclerosis & 1 & 0 & 1 \\
\hline Venous thrombosis & 0 & 1 & 1 \\
\hline \multicolumn{4}{|l|}{ Pulmonary disease } \\
\hline $\mathrm{COPD}^{\mathrm{a}}$ & 0 & 0 & 0 \\
\hline Pulmonary fibrosis & 0 & 1 & 1 \\
\hline Pneumonia & 0 & 4 & 4 \\
\hline Respiratory failure & 0 & 1 & 1 \\
\hline \multicolumn{4}{|l|}{ Others } \\
\hline$C K D^{a}$ & 0 & 4 & 4 \\
\hline Diabetes & 1 & 4 & 5 \\
\hline Local skin infection & 0 & 2 & 2 \\
\hline \multicolumn{4}{|l|}{ ASA classification } \\
\hline $1-2$ & 2 & 11 & 13 \\
\hline $3-4$ & 0 & 7 & 7 \\
\hline \multicolumn{4}{|l|}{ Anesthesia } \\
\hline $\mathrm{GA}^{\mathrm{a}}$ & 1 & 6 & 7 \\
\hline CEA/RBA ${ }^{a}$ & 1 & 12 & 13 \\
\hline LOS (day, median (IQR $\left.\left.{ }^{a}\right)\right)$ & $22 \pm 5.7^{b}$ & $26.5(13.5-81.75)$ & $26(14.5-71.25)$ \\
\hline
\end{tabular}

${ }^{a} C H D$ coronary heart disease, $M I$ myocardial infarction, $C l$ cerebral infarction, $T I A$ Transient ischemic attack, COPD chronic obstructive pulmonary disease, $C K D$ chronic kidney disease, GA general anesthesia, CEA continuous epidural anesthesia, RBA regional block anesthesia, IQR interquartile range

${ }^{b}$ Data shows in mean $\pm S D$, because of only two patients in this group

dysfunction and died of sudden ventricular fibrillation on POD 60 after developing AMI, heart failure, and pneumonia in quick succession. One patient was diagnosed with pulmonary embolism (PE). He developed sudden dyspnea and chest tightness on POD 14 and the diagnosis was confirmed by computed tomographic pulmonary angiography (CTPA). In addition to the aforementioned patients, 2 patients died of sudden dyspnea or cardiac arrest with a suspected diagnosis of PE or AMI.
Only one 82-year-old woman with a history of stroke, CHD, hypertension, and diabetes died of acute CI on POD 2.

Two patients died of other postoperative complications. One with severe respiratory failure caused by worsening pulmonary fibrosis and the other with multiple organ dysfunction syndrome after developing acute $\mathrm{CI}, \mathrm{PE}$, heart failure, renal failure, pneumonia, and gastrointestinal bleeding postoperatively. 
Table 2 Postoperative complications in mortality patients after joint arthroplasty

\begin{tabular}{lll}
\hline & $\begin{array}{l}\text { Elective surgery } \\
\text { TKA }(n=2)\end{array}$ & Non-elective surgery \\
& THA/FHR $(n=18)$ \\
\hline Systemic complications & & \\
Cerebral infarction & $2(100 \%)$ & $2(11 \%)$ \\
Acute Ml & 0 & $6(33 \%)$ \\
Arrhythmia & $1(50 \%)$ & $2(11 \%)$ \\
Acute heart failure & 0 & $3(17 \%)$ \\
PE & 0 & $4(22 \%)$ \\
DVT & $2(11 \%)$ \\
Septic shock & 0 & $7(39 \%)$ \\
Pneumonia & 0 & $10(56 \%)$ \\
Gl ${ }^{\text {a }}$ infection & 0 & $3(17 \%)$ \\
Intracranial infection & 0 & $2(11 \%)$ \\
Urinary tract infection & 0 & $4(22 \%)$ \\
Acute renal failure & 0 & $2(11 \%)$ \\
Bleeding & 0 & $3(17 \%)$ \\
Local complications & & $1(6 \%)$ \\
Deep wound infection & 0 & $1(6 \%)$ \\
Superficial wound infection & 0 & $1(6 \%)$ \\
Wound dehiscence & 0 &
\end{tabular}

${ }^{\mathrm{a} D V T}$ deep venous thrombosis, Gl gastrointestinal

${ }^{b}$ In our study, there are no patients died in hospital after elective THA/FHR or non-elective TKA

\section{Discussion}

To our knowledge, this is the first study to report inpatient mortality rate among Chinese patients undergoing arthroplasty. The overall post-arthroplasty inpatient mortality rate at our center is extremely low, particularly over the last 5 years, which confirms the safety of arthroplasty. However, the mortality rate continues to remain relatively high among those undergoing non-elective procedures.

The cause of death after arthroplasty has changed over time. Previously, cardiovascular disorders including AMI and PE were common in these patients. However, pneumonia has become the most common cause of death over the last 5 years. The perioperative administration of aspirin and low molecular weight heparin has contributed to a reduced incidence of cardiovascular complications.

Owing to the broadened surgical indications, improved surgical skills and the development of highly potent broad-spectrum antibiotics, we have become much more radical to trauma patients with respiratory comorbidities, which has increased the incidence of postoperative pneumonia. Pneumonia is one of the most severe postoperative complications following arthroplasty. The incidence of pneumonia is approximately $0.3 \%$. The mortality risk in patients with postoperative pneumonia is approximately 30-fold higher than that in patients without postoperative pneumonia. Advanced age (especially $\geq 80$ years), smoking, chronic obstructive pulmonary disease (COPD), diabetes, hypertension, and other pulmonary diseases are risk factors for postoperative pneumonia [9]. In our study, 50\% of patients demonstrated symptoms of pneumonia preoperatively with rapid deterioration in symptoms on POD 1 or 2 . However, patients with only risk factors for pneumonia tended to show a later onset of this complication. Therefore, it is recommended that patients with preoperative pneumonia should receive treatment for complete clearance of the infection preoperatively if possible and intraand postoperative antibiotics should be administered

Table 3 distribution of causes of death of inpatient mortality after joint arthroplasty

\begin{tabular}{|c|c|c|c|c|c|c|c|c|}
\hline \multirow[t]{3}{*}{ Cause of death } & \multicolumn{8}{|c|}{ Incidence rate } \\
\hline & \multicolumn{2}{|c|}{$1982-1991$} & \multicolumn{2}{|c|}{ 1992-2001 } & \multicolumn{2}{|l|}{ 2002-2011 } & \multicolumn{2}{|l|}{$2012-2017$} \\
\hline & Elective & Non-elective & Elective & Non-elective & Elective & Non-elective & Elective & Non-elective \\
\hline Any cause & 0 & 2 & 0 & 1 & 2 & 9 & 0 & 6 \\
\hline $\mathrm{Ml}$ & 0 & 0 & 0 & $1(100 \%)$ & 0 & $2(22 \%)$ & 0 & 0 \\
\hline Arrhythmia & 0 & $1(50 \%)$ & 0 & 0 & 0 & 0 & 0 & $1(17 \%)$ \\
\hline PE & 0 & 0 & 0 & 0 & 0 & $1(11 \%)$ & 0 & 0 \\
\hline $\mathrm{Cl}$ & 0 & $1(50 \%)$ & 0 & 0 & $2(100 \%)$ & 0 & 0 & 0 \\
\hline Pneumonia & 0 & 0 & 0 & 0 & 0 & $2(22 \%)$ & 0 & $4(67 \%)$ \\
\hline Abdominal infection & 0 & 0 & 0 & 0 & 0 & $1(11 \%)$ & 0 & 0 \\
\hline Respiratory failure & 0 & 0 & 0 & 0 & 0 & 0 & 0 & $1(17 \%)$ \\
\hline $\mathrm{MODS}^{\mathrm{a}}$ & 0 & 0 & 0 & 0 & 0 & $1(11 \%)$ & 0 & 0 \\
\hline Unclear & 0 & 0 & 0 & 0 & 0 & $2(22 \%)^{b}$ & 0 & 0 \\
\hline All-cause mortality & $0(0 / 136)$ & $3 \%(2 / 65)$ & $0(0 / 468)$ & $0.5 \%(1 / 198)$ & $0.1 \%(2 / 2153)$ & $1.8 \%(9 / 505)$ & $0(0 / 3420)$ & $1.5 \%(6 / 426)$ \\
\hline
\end{tabular}

${ }^{\mathrm{a} M O D S}$ multiple organ dysfunction

${ }^{\mathrm{b}}$ These two patients died from a sudden breath and cardiac arrest, who probably developed PE or AMI 
after sensitivity testing. Patients who show risk factors for pneumonia or other postoperative complications caused immobilization should be treated with a prevention program including oral hygiene care, pulmonary function exercises, early mobility practice, head-of-bed elevation to at least $30^{\circ}$, and sitting up for all meals.

Cardiovascular complications including AMI, heart failure, arrhythmia, and PE are the most common cause of postoperative death after arthroplasty [10]. Bass et al. reported that $13.5 \%$ of patients aged more than 65 years showed elevation serum levels of high-sensitivity cardiac troponin indicating postoperative myocardial ischemia [11]. However, reportedly, the incidence of symptomatic MI was only $0.2-0.7 \%[12,13]$. Thus, a considerable number of patients may show transient asymptomatic myocardial ischemia postoperatively. Advanced age, male sex, a history of cardiac disease (CHD, congestive heart failure, valvular disease), pulmonary circulation disorders, vascular disorders, coagulopathy, diabetes, and anemia as well as fluid and/or electrolyte imbalances are independent risk factors for AMI [14]. The inpatient mortality rate in patients with AMI is approximately $3.4 \%$, which is nearly 40 -fold higher than that in patients without AMI [15]. In our study, all instances of fatal AMI occurred within the first postoperative week, and they occurred even earlier among patients with a history of CHD. Therefore, we emphasized that patients with risk factors should be closely monitored for the symptoms of myocardial ischemia and dynamic electrocardiogram change. Additionally, assessment of myocardial enzyme is warranted within the first week postoperatively. The incidence of symptomatic deep venous thrombosis (DVT) after arthroplasty was $2.1-12.5 \%$ [16, 17]. Without active treatment, approximately $50 \%$ of patients are likely to develop PE, and the condition could be fatal in $5-10 \%$ of these patients [18]. Depending upon the different methods of anticoagulant, the incidence of postoperative PE is $0.16-1.51 \%$ [19]. Our center implemented a postoperative thromboembolism prophylaxis strategy in 2004, which is routinely used presently. Notably, 66.7\% (2/3) of fatal PE occurred in 2003-2004; however, this complication is no longer observed since 2010. Although routine thromboembolism prophylaxis can significantly decrease the incidence of fatal PE, it also increases the risk of postoperative bleeding, hematoma, and periprosthetic infection [20, 21]. Therefore, accurate risk stratification for PE is necessary for decision-making regarding optimal anticoagulant treatment in clinical settings.

The incidence of perioperative stroke is $0.08-0.14 \%$ $[10,22]$. And the inpatient mortality rate in patients who develop stroke postoperatively is approximately $9 \%$ [23]. Advanced age, history of stroke, cardiac valvular disease, peripheral vascular disease, atrial fibrillation, diabetes, and coagulopathy are risk factors for perioperative stroke [22]. Therefore, thorough preoperative risk factors screening is important. Patients presenting with risk factors should undergo close monitoring of blood pressure and large intraoperative fluctuations in blood pressure and/or hypotension should be avoided. Perioperative fluid therapy needs to be optimized to avoid hemoconcentration. In this study, stroke onset occurred in patients within 3 days postoperatively. Therefore, high-risk patients should be closely monitored for early abnormalities in neurological and mental symptoms including delay recovery from anesthesia, a disturbance of consciousness, unexplained unilateral extremities immobility and indifference, euphoria, and/or other psychiatric symptoms. Early recognition, early diagnosis, and prompt treatment can prevent the risk of brain injury and decrease the stroke mortality rate.

\section{Conclusions}

This is the first study to analyze the inpatient mortality rates in Chinese patients undergoing arthroplasty. We conclude that arthroplasty is a safe and effective procedure associated with a relatively low inpatient mortality in China. And inpatient mortality does not increase as the growing surgery volume due to improvement of perioperative management. However, patients presenting with risk factors and those undergoing non-elective procedures demonstrated a relatively high incidence of postoperative complications, particularly pneumonia. Thorough preoperative assessment, close perioperative monitoring and the institution of optimal individualized prophylaxis are warranted to reduce inpatient mortality.

\section{Abbreviations \\ AMI: Acute myocardial infarction; ASA: American Society of Anesthesiologists; CEA: Continuous epidural anesthesia; CHD: Coronary heart disease; Cl: Cerebral infarction; CKD: Chronic kidney disease; COPD: Chronic obstructive pulmonary disease; CTPA: Computed tomographic pulmonary angiography; DVT: Deep venous thrombosis; FHR: Femoral head replacement; GA: General anesthesia; GI: Gastrointestinal; ICU: Intensive care unit; IQR: Interquartile range; LOS: Length of stay; MODS: Multiple organ dysfunction; PE: Pulmonary embolism; POD: Post operation day; RBA: Regional block anesthesia; THA: Total hip arthroplasty; TIA: Transient ischemic attack; TKA: Total knee arthroplasty}

\section{Acknowledgements}

We thank Dr. Yanyan Bian, Shuai Xiang, Zeng Li, and all the other colleges who helped in collection clinical information. We also thank Jianyi Wang for improving the English language of our article.

\section{Authors' contributions}

$J L, J J, W Q, G Q$, and $X W$ are orthopedic surgeons who performed these surgeries. $X W$ designed this study and $Y Z$ analyzed and interpreted the patient data. All authors read and approved the final manuscript.

Funding

No funding supported this study.

Availability of data and materials

All data generated or analyzed during this study are included in this published article. 


\section{Ethics approval and consent to participate}

This is a retrospective study, and it was approved by the Ethics Committee of Peking Union Medical College Hospital, Peking Union Medical College, and Chinese Academy of Medical Sciences.

\section{Consent for publication}

Not applicable.

\section{Competing interests}

The authors declare that they have no competing interests.

Received: 3 January 2019 Accepted: 7 June 2019

Published online: 17 June 2019

\section{References}

1. Michet CJ 3rd, Schleck CD, Larson DR, Maradit Kremers H, Berry DJ, Lewallen DG. Cause-specific mortality trends following total hip and knee arthroplasty. J Arthroplast. 2017;32:1292-6.

2. Lisenda L, Mokete L, Mkubwa J, Lukhele M. Inpatient mortality after elective primary total hip and knee joint arthroplasty in Botswana. Int Orthop. 2016; 40:2453-5.

3. Lalmohamed A, Vestergaard P, de Boer A, Leufkens HG, van Staa TP, de Vries $F$. Changes in mortality patterns following total hip or knee arthroplasty over the past two decades: a nationwide cohort study. Arthritis Rheum. 2014:66:311-8.

4. Memtsoudis SG, Pumberger M, Ma Y, Chiu YL, Fritsch G, Gerner P, et al. Epidemiology and risk factors for perioperative mortality after total hip and knee arthroplasty. J Orthop Res. 2012;30:1811.

5. Kirksey M, Chiu YL, Ma Y, Della Valle AG, Poultsides L, Gerner P, et al. Trends in in-hospital major morbidity and mortality after total joint arthroplasty: United States 1998-2008. Anesth Analg. 2012;115:321-7.

6. Singh JA, Kundukulam J, Riddle DL, Strand V, Tugwell P. Early postoperative mortality following joint arthroplasty: a systematic review. J Rheumatol. 2011:38:1507.

7. Singh JA, Lewallen DG. Ninety-day mortality in patients undergoing elective total hip or Total knee arthroplasty. J Arthroplast. 2012;27:1417-6.

8. Prokop A, Chmielnicki M. Hemiprosthesis for femoral neck fractures in the elderly: a retrospective study of 319 patients. Arch Trauma Res. 2016;5: e33335.

9. Bohl DD, Saltzman BM, Sershon RA, Darrith B, Okroj KT, Della Valle CJ. Incidence, risk factors, and clinical implications of pneumonia following total hip and knee arthroplasty. J Arthroplast. 2017;32:1991-5.

10. Pulido L, Parvizi J, Macgibeny M, Sharkey PF, Purtill JJ, Rothman RH, et al. In hospital complications after total joint arthroplasty. J Arthroplast. 2008;23: 139-7.

11. Bass AR, Rodriguez T, Hyun G, Santiago FG, Kim JI, Woller SC, et al. Myocardial ischemia after hip and knee arthroplasty: incidence and risk factors. Int Orthop. 2015;39:2011-6.

12. Pedersen $A B$, Mehnert F, Sorensen HT, Emmeluth C, Overgaard S, Johnsen $\mathrm{SP}$. The risk of venous thromboembolism, myocardial infarction, stroke, major bleeding and death in patients undergoing total hip and knee replacement: a 15-year retrospective cohort study of routine clinical practice. Bone Joint J. 2014;96-B:479-7.

13. Thornqvist C, Gislason GH, Kober L, Jensen PF, Torp-Pedersen C, Andersson C. Body mass index and risk of perioperative cardiovascular adverse events and mortality in 34,744 danish patients undergoing hip or knee replacement. Acta Orthop. 2014;85:456-7.

14. Menendez ME, Memtsoudis SG, Opperer M, Boettner F, Gonzalez Della Valle A. A nationwide analysis of risk factors for in-hospital myocardial infarction after total joint arthroplasty. Int Orthop. 2015;39:777-10.

15. Illingworth KD, El Bitar YF, Banerjee D, Scaife SL, Saleh KJ. Inpatient mortality after primary total hip arthroplasty: analysis from the National Inpatient Sample Database. J Arthroplast. 2015;30:369-5.

16. Nillius AS, Nylander G. Deep vein thrombosis after total hip replacement: a clinical and phlebographic study. Br J Surg. 1979;66:324-3.

17. Dahl OE, Gudmundsen TE, Haukeland L. Late-occurring clinical deep vein thrombosis in joint-operated patients. Acta Orthop Scand. 2000;71:47-4.

18. Eikelboom JW, Quinlan DJ, Douketis JD. Extended-duration prophylaxis against venous thromboembolism after total hip or knee replacement: a meta-analysis of the randomized trials. Lancet. 2001;358:9-7.
19. Freedman KB, Brookenthal KR, Fitzgerald RH Jr, Williams S, Lonner JH. A meta-analysis of thromboembolic prophylaxis following elective total hip arthroplasty. J Bone Joint Surg Am. 2000;82-A:929-10.

20. Guijarro R, Montes J, San Román C, Arcelus Jl, Barillari G, Granero X, et al. Venous thromboembolism and bleeding after total knee and hip arthroplasty. Findings from the Spanish National Discharge Database. Thromb Haemost. 2011;105:610-6.

21. Parvizi J, Ghanem E, Joshi A, Sharkey PF, Hozack WJ, Rothman RH. Does "excessive" anticoagulation predispose to periprosthetic infection? J Arthroplast. 2007;22:24-5.

22. Menendez ME, Greber EM, Schumacher CS, Lowry Barnes C. Predictors of acute ischemic stroke after total knee arthroplasty. J Surg Orthop Adv. 2017; 26:148-6.

23. Rasouli MR, Tabatabaee RM, Maltenfort MG, Chen AF. Acute stroke after total joint arthroplasty: a population-based trend analysis. J Clin Anesth. 2016;34:15-6.

\section{Publisher's Note}

Springer Nature remains neutral with regard to jurisdictional claims in published maps and institutional affiliations.
Ready to submit your research? Choose BMC and benefit from:

- fast, convenient online submission

- thorough peer review by experienced researchers in your field

- rapid publication on acceptance

- support for research data, including large and complex data types

- gold Open Access which fosters wider collaboration and increased citations

- maximum visibility for your research: over $100 \mathrm{M}$ website views per year

At BMC, research is always in progress.

Learn more biomedcentral.com/submissions 